\title{
Mathematical models in physiology
}

\author{
By David Gavaghan ${ }^{1, *}$, Alan Garny ${ }^{2}$, Philip K. Maini $^{3}$ \\ ANd Peter KOHL ${ }^{2}$ \\ ${ }^{1}$ Oxford University Computing Laboratory, Wolfson Building, Parks Road, \\ Oxford $O X 13 Q D, U K$ \\ ${ }^{2}$ Cardiac Mechano-Electric Feedback Group, Department of Physiology, \\ Anatomy and Genetics, Sherrington Building, Parks Road, \\ Oxford OX1 3PT, UK \\ ${ }^{3}$ Centre for Mathematical Biology, Mathematical Institute, 24-29 St Giles', \\ Oxford $O X 13 L B, U K$
}

Computational modelling of biological processes and systems has witnessed a remarkable development in recent years. The search-term (modelling OR modeling) yields over 58000 entries in PubMed, with more than 34000 since the year 2000: thus, almost twothirds of papers appeared in the last 5-6 years, compared to only about one-third in the preceding 5-6 decades.

The development is fuelled both by the continuously improving tools and techniques available for bio-mathematical modelling and by the increasing demand in quantitative assessment of element inter-relations in complex biological systems. This has given rise to a worldwide public domain effort to build a computational framework that provides a comprehensive theoretical representation of integrated biological function-the Physiome.

The current and next issues of this journal are devoted to a small sub-set of this initiative and address biocomputation and modelling in physiology, illustrating the breadth and depth of experimental data-based model development in biological research from sub-cellular events to whole organ simulations.

Keywords: physiome; quantitative biology; systems biology; theoretical biology; integrative physiology

\section{Introduction}

The May and June issues of the Philosophical Transactions of the Royal Society are devoted to biocomputation and modelling in physiology. They form a focussed issue, which has its roots in an international workshop on the same topic, held by The Physiological Society at Oxford from 30 September to 2 October 2004.

The workshop was organized to coincide with the retirement of Professor Denis Noble, CBE, FRS, FRCP (hon.), from the British Heart Foundation Burdon Sanderson Chair at the University of Oxford. It commenced with the

* Author for correspondence (david.gavaghan@comlab.ox.ac.uk).

One contribution of 13 to a Theme Issue 'Biomathematical modelling I'. 
Physiological Society's esteemed Hodgkin-Huxley-Katz Prize Lecture, delivered by Professor Noble. The awardee and speaker was introduced by no other than Nobel laureate Professor Sir Andrew Huxley - a beautiful illustration of the origins of a field that is now ubiquitously present in the life sciences: mathematical modelling. This field owes a depth of gratitude to these preeminent scientists who, commencing with the 'paradigm' physiological model of nerve excitation of Hodgkin \& Huxley (1952) and the first cardiac cell model of Noble (1960), laid the foundations for development of the current, quantitative approach to computational modelling of biological systems, which is thoroughly based on experimental data, and aims to make experimentally verifiable predictions.

During the half-century since those beginnings, and particularly over the last decade, there has been a rapid expansion in both computing resources and detailed experimental data. As a result, work towards developing a 'theoretical biology' (Noble 2002a) has progressed at a continuously increasing pace, as can be seen in the increasing popularity of 'systems biology'. As Denis Noble pointed out, although there is as yet no clear definition of the term systems biology (Noble 2005), a consensus is emerging that it represents an approach to biological research that attempts to provide a quantitative theoretical description of the manner in which biological and physiological function emerges from interactions between system components across multiple spatial and temporal scales. It is necessarily both interdisciplinary and fully grounded within experimental data, and as such can be seen to reflect the pioneering spirit of Huxley, Hodgkin, Noble and others.

This extensive, double-issue of the Philosophical Transactions of the Royal Society features contributions at the workshop and serves as an illustration of both the depth and breadth of mathematical modelling in quantitative physiological research. Given Denis Noble's enduring work in the area of detailed cardiac cell modelling, just over half of the contributions are devoted to the heart, while the remainder describe theoretical studies in areas as diverse as the modelling of wound healing, cancer and renal function. Rather than arranging contributions topically, this special issue follows a spatial scale, from the sub-cellular and cellular levels, through to tissue, organ and organism, followed by reviews crossing multiple scales and reports on emerging generic infrastructures and tools to support physiological modelling.

\section{Focus on sub-cellular, cellular and inter-cellular behaviour}

It is at the sub-cellular and cellular levels that Denis Noble's own most influential contributions occurred, starting with his Nature paper in 1960 (Noble 1960), which introduced the first mathematical and computational model of a cardiac Purkinje fibre cell, right through to the present day with his 2004 paper, in collaboration with ten Tusscher and Panfilov, presenting a detailed model of human ventricular cardiomyocyte activity (ten Tusscher et al. 2004). In this special issue, papers devoted to cell modelling demonstrate in detail the progress that has been made in this area, reflecting the current state-of-the-art and highlighting primary research foci within the field. These papers necessarily focus in some detail on a particular aspect of cardiac cell functionality, attempting to derive mathematical 
relationships, predominantly in the form of Hodgkin-Huxley type ordinary differential equations, which support computer simulations that reproduce experimental data, increasingly with predictive power (Noble 2002b).

At the sub-cellular level, Iribe, in a joint contribution with Denis Noble and Peter Kohl, considers a model of cardiac calmodulin-dependent kinase II, hypothesizing that the slow inactivation of this enzyme and its modulatory effect on $\mathrm{Ca}^{2+}$ handling in the sarcoplasmic reticulum are key to understanding different interval-force relations (Iribe et al. 2006). Modelling at the sub-cellular level has advanced to an equally impressive level in other biological tissues, partially guided by direct knowledge transfer from cardiac to other cell models, as can be illustrated by the paper by Youm et al., who simulate non-cardiac pacemaker activity in gastrointestinal tract smooth muscle (Youm et al. 2006). Going even deeper in the hierarchy of sub-cellular processes, Bernard et al. model transcriptional feedback and its role in developmental biology using the example of the transcriptional repressor Hes1, which periodically changes its expression pattern in the presomitic mesoderm (Bernard et al. 2006). Models of this type are becoming an increasingly important focus of the mathematical biology literature, as the research community attempts to grapple with the complexities of gene regulation networks.

At the cellular level, five papers consider various models of ventricular myocyte activity. Crampin et al. focus on the role of acidosis in cardiac electrophysiology and excitation-contraction coupling, reviewing the experimental findings and presenting a modelling study that establishes the contribution of the different effects to altered $\mathrm{Ca}^{2+}$ transients during acidosis (Crampin et al. 2006). Pásek et al. consider the role of cardiac T-tubules in the physiological modulation of electrical and contractile activity through development of a mathematical model of ventricular cardiomyocytes in which the cardiac transverse axial tubular system is described as a single compartment, allowing them to demonstrate the effects of this system on $\mathrm{Ca}^{2+}$ and $\mathrm{K}^{+}$handling (Pásek et al. 2006). Fink et al. gain an improved understanding of the role of inwardly rectifying $\mathrm{K}^{+}$currents in repolarization of the action potential in cardiac muscle by assessing three recently described mathematical models of human ventricular myocytes (Fink et al. 2006). Leem et al. conduct a detailed comparison between experimental and simulation data in modelling the $\mathrm{Ca}^{2+}$-activated $\mathrm{Cl}^{-}$current of cardiomyocytes (Leem et al. 2006). Finally, Terashima et al. consider the modelling of $\mathrm{Cl}^{-}$homeostasis and volume regulation of cardiac cells (Terashima et al. 2006).

At the intercellular level, Marée et al. confirm the role of single cell models as important building blocks for simulations that move towards higher levels of structural and functional integration, by addressing the cell-to-cell interactions in the initial events underlying the onset of type 1 diabetes (Marée et al. 2006).

\section{Tissue level models}

As we move up the spatial scales within biology, it becomes necessary to integrate cellular models into more complex descriptions at the tissue level. A common approach to this problem is to invoke a continuum hypothesis, by assuming that a sufficient number of cells are present to allow an average, 
continuous description of variables of interest to be made across the whole tissue. This approach is taken in most of the models described in this section, with six of the eight papers devoted to various aspects of cardiac tissue behaviour. In the heart, a main area of interest is the propagation of electrical activity and its effect on function. Two of these contributions (Biktashev et al. 2006; Steinberg et al. 2006) harness the power of mathematical analysis to abstract key features of the system through the use of greatly simplified mathematical descriptions, which capture certain aspects of fundamentally important dynamics of a biological system (in this case its excitability) without the need to incorporate much of the biological detail. In another application of cardiac model-derived 'know-how', Benson et al. use similar analytical tools to explore how previous bifurcation analysis of myocardial excitability parameter values can be extended to uterine tissue (Benson et al. 2006).

Bourne et al. and Kuo and Trayanova use mathematical models to investigate clinically relevant questions and derive a more detailed and comprehensive mathematical description of cellular level effects, including ionic currents (the Beeler \& Reuter (1977) model in Bourne et al. (2006); the Ramirez et al. (2000) model in Kuo \& Trayanova (2006)) coupled to bidomain equations that describe the propagation of electrical activity both in the intracellular and extracellular spaces. This allows Bourne et al. to simulate optimized electric shock application protocols for defibrillation of mammalian heart tissue (Bourne et al. 2006), while Kuo and Trayanova consider the effects of heterogeneity in cardiac action potential morphology in the context of atrial fibrillation (Kuo \& Trayanova 2006). Solovyova et al., on the other hand, use mechanically connected onedimensional models of myocardial tissue to show that the sequence of cardiac electrical activation is a key factor in spatio-temporal organization of the heart (Solovyova et al. 2006).

Finally, two papers investigate non-cardiac tissue models. First, McDougal et al. introduce a multi-scale modelling approach to the problem of wound healing and address the role of collagen alignment, a key determinant of scar tissue formation properties (McDougal et al. 2006). Second, Lu and Wang present initial findings from their work on quantifying osmotic pressure in the extracellular matrix and on its contribution to the viscoelastic characteristics of soft tissues during deformation (Lu \& Wang 2006).

\section{Organ and organism}

At the organ and organism level, complexity of computational (and experimental) models increases rapidly. In order to handle this problem, the multitude of interacting processes and components must be assessed for inclusion into, or elimination from, mathematical representation of biological behaviour. Different authors have taken different approaches, but applied (i.e. experimentally testable) work seems to follow the pattern that, once the research question has been determined experimentally, the mathematical models are developed to maximally reproduce relevant behaviour with minimal complexity. This process of selection and reduction is, of course, difficult and usually requires a continuous iteration between experimental and theoretical model application. 
Each contribution within this section takes a rather different approach to the modelling phase. Bassingthwaighte et al. present a generic approach to generalized blood-tissue exchange modelling in whole organ studies (Bassingthwaighte et al. 2006). This extends previous work by increasing the number of cell types and accounting for nonlinear competitive processes for transport, binding and reaction, with a demonstration of the authors' ultimate goal of being able to fit multiple datasets simultaneously.

Schaller and Meyer-Herman develop a continuum model of the growth of multi-cellular tumour spheroids, which includes the effects of oxygen and glucose, with model parameters determined by fitting to experimental growth curves (Schaller \& Meyer-Herman 2006). The model is compared to a discrete agentbased approach, developed previously by the same authors, with the conclusion that the continuous model gives similarly accurate results, but at significantly greater computational efficiency. This model makes the mathematically convenient assumption of spherical symmetry, allowing a one-dimensional problem to be solved with the concomitant savings in simulation time.

The issue of computational efficiency (and even tractability) becomes increasingly important as one moves into three dimensions, as is necessitated by the modelling of realistic physiological geometries or targeting the systems level (Garny et al. 2005). The work of Seemann et al. is a good example of how advanced the field of heart modelling has become in this regard (Seemann et al. 2006). They present an anatomically based model of the human atria that incorporates both structural and electrophysiological heterogeneities. This is combined with very detailed models of the various cell types to provide, via computer simulation, insight into the mechanisms underlying the genesis and conduction of electrical excitation within the atria. Simulation of $1 \mathrm{~s}$ cardiac activity required $37 \mathrm{~h}$ of processor time on a 20-node cluster, making it clear that a detailed parameter search in such comprehensive models is still prohibitively expensive and that there is much room for research into development of more computationally efficient solution methods for very complex physiological problems.

At the system level, most authors opt to greatly simplify the initial model in order to gain insight into the underlying physiological system. This is the approach taken by Shim et al. in their multi-scale model of the circulation (Shim et al. 2006). This model combines a detailed representation of a ventricular cell with lumped phenomenological models of the circulation, allowing the authors to explore how blood pressure dynamics in the ventricle and aorta are related to cellular processes. Their simulations reproduce typical characteristics of cardiac mechanics, such as the pressure-volume relationship, stroke volume and the effect of the increased maximum free $\mathrm{Ca}^{2+}$ concentration on cardiovascular haemodynamics.

\section{Infrastructure challenges and reviews}

Three contributions address different aspects of the computational complexity and information technology infrastructure requirements that arise from detailed physiological modelling, particularly in higher dimensions. Pitt-Francis et al. describe their experience in taking an existing heart modelling package 
(Garny et al. 2003), designed for use on a PC, and porting it so that the computational core of the software can be run on state-of-the-art high performance computers using the grid infrastructure that is currently being developed in the UK via the National e-Science Programme (Pitt-Francis et al. 2006). Ribba et al. focus on the provision of generic databases, designed to support physiological modelling process by making quantitative and structural information, derived from both experiment and mathematical models, more readily available to research scientists over the Web (Ribba et al. 2006). This development of a suitable information technology infrastructure to support the sharing of scientific data and information is becoming increasingly crucial to the success of research in the sciences, as more and more studies involve largescale international collaboration. The implications of the changes being brought about by this trend, together with the increasingly interdisciplinary nature of scientific research, is discussed from a sociological, organizational and technological perspective by Welsh et al. (2006). The authors draw on the literature describing the impact of previous technological changes to delineate the challenges that are likely to arise in research in the life and clinical sciences.

Finally, two reviews illustrate the development of separate fields over the course of several decades. Pinter and Shohet review 40 years of research into the modelling of the kidney, before presenting their theory on the role of hyaluronate in the inner medullary concentrating process (Pinter \& Shohet 2006). Byrne et al. present a timely review of the modelling of cancer dynamics, focusing on the inherent complexity involved in modelling a disease in which a variety of factors, many of which may still be unknown, interact over a wide range of spatial and temporal scales (Byrne et al. 2006). They point out that, although huge data sets relating to the different scales are available, these data do not always reveal the mechanisms underpinning observed phenomena. They further show the potential of mathematics for interpreting this data, by presenting case studies that illustrate the types of insight that theoretical models of solid tumour growth may yield. This paper concludes with an illustration of the benefits that result from close collaboration between experimentalists and theoreticians (considering a novel anti-cancer therapy), and takes us back to the key point made by Huxley and Noble: physiological modelling must be thoroughly grounded in experimental data if it is to be successful.

The editors hope that the present collection of works will convey the spirit of the Oxford workshop on Biocomputation and Modelling in Physiology, by illustrating the breadth of the field, highlighting achievements made already, and demonstrating the enormity of the task that faces us in attempting to develop a quantitative systems biology - truly a Grand Challenge both for computing and life sciences.

The workshop on Biocomputation and Modelling in Physiology would not have been possible without the vision and support of the UK Physiological Society. We further thank The British Heart Foundation, Novartis, and Pfizer for their generous financial support in making this the successful event it was. Last, but not least, the editors would like to acknowledge Charlotte Smith who has been responsible for the administrative coordination of this focussed issue. 


\section{References}

Bassingthwaighte, J. B., Raymond, G. M., Ploger, J. D., Schwartz, L. M. \& Bukowski, T. R. 2006 GENTEX, a general multiscale model for in vivo tissue exchanges and intraorgan metabolism. Phil. Trans. R. Soc. A 364. (doi:10.1098/rsta.2006.1779)

Beeler, G. W. \& Reuter, H. 1977 Reconstruction of the action potential of ventricularmyocardial fibres. J. Physiol. 268, 177-210.

Benson, A. P., Clayton, R. H., Holden, A. V., Kharche, S. \& Tong, W. C. 2006 Endogenous driving and synchronization in cardiac and uterine virtual tissues: bifurcations and local coupling. Phil. Trans. R. Soc. A 364, 1313-1327. (doi:10.1098/rsta.2006.1772)

Bernard, S., Branka, C., Pujo-Menjouet, L., Mackey, M. C. \& Herzel, H. 2006 Modelling transcriptional feedback loops: the role of Gro/TLE1 in Hes1 oscillations. Phil. Trans. R. Soc. A 364, 1155-1170. (doi:10.1098/rsta.2006.1761)

Biktashev, I. V., Simitev, R. D., Suckley, R. \& Biktashev, V. N. 2006 Asymptotic properties of mathematical models of excitability. Phil. Trans. R. Soc. A 364, 1283-1298. (doi:10.1098/rsta. 2006.1770)

Bourne, D. W., Maleckar, M. M., Rodriguez, B. \& Trayanova, N. 2006 Mechanistic inquiry into the effect of rapid pacing on the upper limit of vulnerability. Phil. Trans. R. Soc. A 364. (doi:10. 1098/rsta.2006.1775)

Byrne, H. M., Alarcon, T., Owen, M. R., Webb, S. D. \& Maini, P. K. 2006 Modelling aspects of cancer dynamics: a review. Phil. Trans. R. Soc. A 364. (doi:10.1098/rsta.2006.1786)

Crampin, E., Smith, N., Langham, A. E., Clayton, R. H. \& Orchard, C. H. 2006 Acidosis in models of cardiac ventricular myocytes. Phil. Trans. R. Soc. A 364, 1171-1186. (doi:10.1098/rsta.2006. 1763)

Fink, M., Giles, W. R. \& Noble, D. 2006 Contributions of inwardly rectifying K+ currents to repolarization assessed using mathematical models of human ventricular myocytes. Phil. Trans. R. Soc. A 364, 1207-1222. (doi:10.1098/rsta.2006.1765)

Garny, A., Kohl, P. \& Noble, D. 2003 Cellular open resource (COR): a public CellML based environment for modelling biological function. Int. J. Bifurcat. Chaos 13, 3579-3590. (doi:10. 1142/S021812740300882X)

Garny, A., Noble, D. \& Kohl, P. 2005 Dimensionality in cardiac modelling. Prog. Biophys. Mol. Biol. 87, 47-66. (doi:10.1016/j.pbiomolbio.2004.06.006)

Hodgkin, A. L. \& Huxley, A. F. 1952 A quantitative description of membrane current and its application to conduction and excitation in nerve. J. Physiol. 117, 500-544.

Iribe, G., Kohl, P. \& Noble, D. 2006 Modulatory effect of calmodulin dependent kinase II (CaMKII) on sarcoplasmic reticulum $\mathrm{Ca}^{2+}$ handling and interval-force relations: a modelling study. Phil. Trans. R. Soc. A 364, 1107-1133. (doi:10.1098/rsta.2006.1758)

Kuo, S. R. \& Trayanova, N. A. 2006 Action potential morphology heterogeneity in the atrium and its effect on atrial reentry. Phil. Trans. R. Soc. A 364. (doi:10.1098/rsta.2006.1776)

Leem, C. H., Kim, W. T., Ha, J. M., Lee, Y. J., Seong, H. C., Choe, H., Jang, Y. J., Youm, J. B. \& Earm, Y. E. 2006 Simulation of $\mathrm{Ca}^{2+}$-activated $\mathrm{Cl}^{-}$current of cardiomyocytes in rabbit pulmonary vein: implications of subsarcolemmal $\mathrm{Ca}^{2+}$ dynamics. Phil. Trans. R. Soc. A 364, 1223-1243. (doi:10.1098/rsta.2006.1766)

Lu, Y. \& Wang, W. 2006 Effects of osmotic pressure in extracellular matrix on tissue deformation. Phil. Trans. R. Soc. A 364. (doi:10.1098/rsta.2006.1778)

Marée, A. F., Kublik, R., Finegood, D. T. \& Edelstein-Kehshet, L. 2006 Modelling the onset of type 1 diabetes: can impaired macrophage phagocytosis make the difference between health and disease? Phil. Trans. R. Soc. A 364, 1267-1282. (doi:10.1098/rsta.2006.1769)

McDougall, S., Dallon, J., Sherratt, J. \& Maini, P. 2006 Fibroblast migration and collagen deposition during dermal wound healing: mathematical modelling and clinical implications. Phil. Trans. R. Soc. A 364. (doi:10.1098/rsta.2006.1773)

Noble, D. 1960 Cardiac action and pacemaker potentials based on the Hodgkin-Huxley equations. Nature 188, 495-497. 
Noble, D. $2002 a$ The rise of computational biology. Nat. Rev. Mol. Cell Biol. 3, 460-463. (doi:10. $1038 / \mathrm{nrm} 810)$

Noble, D. $2002 b$ Modeling the heart-from genes to cells to the whole heart. Science 295, 1678-1682. (doi:10.1126/science.1069881)

Noble, D. 2005 Oxford focused meeting. Physiol. news 58.

Pásek, M., Šimurda, J. \& Christé, G. 2006 The functional role of cardiac T-tubules explored in a model of rat ventricular myocytes. Phil. Trans. R. Soc. A 364, 1187-1206. (doi:10.1098/rsta. 2006.1764)

Pinter, G. G. \& Shohet, J. L. 2006 Two fluid compartments in the renal inner medulla. A view through the keyhole of the concentrating process. Phil. Trans. R. Soc. A 364. (doi:10.1098/rsta. 2006.1774)

Pitt-Francis, J., Garny, A. \& Gavaghan, D. 2006 Enabling computer models of the heart for highperformance computers and the grid. Phil. Trans. R. Soc. A 364. (doi:10.1098/rsta.2006.1783)

Ramirez, R., Nattel, S. \& Courtemanche, M. 2000 Mathematical analysis of canine atrial action potentials: rate, regional factors, and electrical remodelling. Am. J. Physiol. 279, H1767-H1785.

Ribba, B., Tracqui, P., Boix, J., Boissel, J. \& Randall Thomas, S. 2006 QxDB: a generic database to support mathematical modeling in biology. Phil. Trans. R. Soc. A 364. (doi:10.1098/rsta. 2006.1784)

Schaller, G. \& Meyer-Herman, M. 2006 Continuum versus discrete model: a comparison for multicellular tumour spheroids. Phil. Trans. R. Soc. A 364. (doi:10.1098/rsta.2006.1780)

Seemann, G., Höper, C., Sachse, F. B., Dössell, O., Holden, A. V. \& Zhang, H. 2006 Heterogeneous 3D anatomical and electrophysiological model of human atria. Phil. Trans. R. Soc. A $\mathbf{3 6 4}$. (doi:10.1098/rsta.2006.1781)

Shim, E. B., Leem, C. H., Abe, Y. \& Noma, A. 2006 A new multi-scale simulation model of the circulation: from cells to system. Phil. Trans. R. Soc. A 364. (doi:10.1098/rsta.2006.1782)

Solovyova, O., Katsnelson, L. B., Konovalov, P., Lookin, O., Moskvin, A. S., Protseko, Y. L., Vikulova, N., Kohl, P. \& Markhasin, V. S. 2006 Activation sequence as a key factor in spatiotemporal optimization of myocardial function. Phil. Trans. R. Soc. A 364. (doi:10.1098/rsta. 2006.1777)

Steinberg, B. E., Glass, L., Shrier, A. \& Bub, G. 2006 The role of heterogeneities and intercellular coupling in wave propagation in cardiac tissue. Phil. Trans. R. Soc. A 364, 1299-1311. (doi:10. 1098/rsta.2006.1771)

ten Tusscher, K. H., Noble, D., Noble, P. J. \& Panfilov, A. V. 2004 A model for human ventricular tissue. Am. J. Physiol. 286, 1573-1589.

Terashima, K., Takeuchi, A., Sarai, N., Matsuoka, S., Shim, E. B., Leem, C. H. \& Noma, A. 2006 Modelling Cl- homeostasis and volume regulation of the cardiac cell. Phil. Trans. R. Soc. A 364, 1245-1265. (doi:10.1098/rsta.2006.1767)

Welsh, E., Jirotka, M. \& Gavaghan, D. 2006 Post-genomic science: multidisciplinary and largescale collaborative research and its organisational and technological implications for the scientific research process. Phil. Trans. R. Soc. A 364. (doi:10.1098/rsta.2006.1785)

Youm, J. B., Kim, N., Han, J., Kim, E., Joo, H., Leem, C. H., Goto, G., Noma, A. \& Earm, Y. E. 2006 A mathematical model of pacemaker activity recorded from mouse small intestine. Phil. Trans. R. Soc. A 364, 1135-1154. (doi:10.1098/rsta.2006.1759) 